\title{
Indagación en el laboratorio de física de secundaria: ¿cuáles serían las mejores condiciones para hacer kayak?
}

\author{
Sabela Otero Rial y Beatriz Crujeiras Pérez. Universidad de Santiago de Compostela. \\ Recepción: 29 de julio de 2016 | Revisión: 6 de septiembre de 2016 | Aceptado: 17 octubre de 2016 \\ Correspondencia: Beatriz Crujeiras Pérez | Email: beatriz.crujeiras@usc.es \\ Citar: Otero, S. y Crujeiras, B. (2016). Indagación en el laboratorio de física de secundaria: ¿cuáles serían las mejores condiciones \\ para hacer kayak?. ReiDoCrea, 5, 235-246.
}

Resumen: Los cambios sociales producidos en las últimas décadas han llevado al requerimiento de una modificación en la metodología de enseñanza hacia una fundamentación en la autonomía del alumnado y la contextualización del aprendizaje. Por este motivo cobra especial relevancia el papel del laboratorio y, sobre todo, de enfoques como el aprendizaje de las ciencias basado en la indagación. Teniendo en cuenta que, en general, las investigaciones muestran que la enseñanza a través de la indagación produce resultados positivos para el aprendizaje del alumnado, el objetivo de este estudio es analizar los desempeños del alumnado durante la realización de una actividad de indagación guiada en el laboratorio. El estudio se enmarca en la investigación cualitativa y se centra en el análisis de las producciones del alumnado a través de una rúbrica. Entre los resultados recogidos destaca la existencia de ciertas operaciones de indagación que entrañan una mayor complicación para los y las estudiantes, así como la dificultad en la adaptación al nuevo enfoque. Sin embargo, se puede observar también que la implantación de este tipo de experiencias fomenta el interés y favorece el desarrollo de la competencia científica.

Palabras clave: Laboratorio escolar | Física

Inquiry in the high school physics laboratory: which conditions would be the best to practice kayaking?

\begin{abstract}
The social changes produced in the last decades have ended in the requirement of a change in the teaching methods aimed at developing students' autonomy in learning and contextualization practices. For this reason the role of the laboratory in Science learning becomes very relevant, in particular approaches like inquiry-based learning. Taking into account that, research in the literature shows that teaching through inquiry produces positive results in students' learning, the goal of this study is to analyse the students' performances during the performance of a guided inquiry task in the laboratory. The study draws on qualitative research and the focus is on the analysis of students' productions by means of a rubric. The main results highlight the existence of certain inquiry operations that students find difficult as well as the adaptation to the new approach. However, it can be also observed that the implementation of this type of experiences promotes students' interest in learning and enables the development of scientific competence.
\end{abstract}

Key words: School laboratorios | Physics

\section{Introducción}

La educación científica se enmarca en una sociedad marcada por los avances científico-tecnológicos y por la rápida obsolescencia del conocimiento, por lo que se demandan ciudadanos competentes científicamente y capacitados para una adaptación a un cambio constante (Tortosa, 2013). Estas circunstancias requieren de 
una modificación en la metodología de enseñanza para distanciarla de aquella basada en la memorización (Álvarez, Arias, Pérez e Serrallé, 2013). Debido a esto, las principales recomendaciones en didáctica de las ciencias proponen que la enseñanza ha de estar fundamentada en la autonomía del alumnado, la interdisciplinariedad, la comunicación y cooperación y en un enfoque contextualizado (García-Carmona, 2009). Por este motivo, en las últimas décadas, el uso del laboratorio ha adoptado un papel central en la enseñanza de las ciencias (Hofstein y Lunetta, 2003) por permitir la introducción del trabajo experimental en el aula (Cortel et al, 2004).

Los trabajos experimentales en el aula pueden clasificarse de distintas formas. En el artículo de Windschitl (2003) se propone una clasificación basada en la aportación por parte del docente de una pregunta, una respuesta a dicha pregunta y el procedimiento para resolverla, como se resume en la tabla 1.

Tabla 1. Clasificación de los trabajos de laboratorio en función de la pregunta, la respuesta y el procedimiento (tomado de Windchitl, 2003)

\begin{tabular}{llll}
\hline Tipo de trabajo & Pregunta & Procedimiento & Respuesta \\
\hline Experiencias de confirmación & Docente & Docente & Docente \\
Indagación estructurada & Docente & Docente & Alumno \\
Indagación guiada & Docente & Alumno & Alumno \\
Indagación abierta o independiente & Alumno & Alumno & Alumno \\
\hline
\end{tabular}

En el presente estudio el enfoque escogido es la indagación guiada, en el cual el único de los tres factores aportado por el docente es el problema que se va a investigar, pero la resolución del mismo depende en su totalidad del estudiante (Bell, Smetana y Binns, 2005).

La indagación en el laboratorio es la acción de construir y comprobar el conocimiento a través de una investigación o búsqueda (Barrow, 2006; Ferrés, Marbà y Sanmartí, 2015). Esta estrategia de aprendizaje se basa en la capacidad del alumnado de investigar y en el constructivismo, tomando como punto de partida los conocimientos previos del alumnado (García-Carmona, 2011). La indagación guiada supone un proceso activo de aprendizaje que capta la atención de los estudiantes a través de un amplio rango de actividades (Anderson, 2002) fundamentadas en preguntas (Artigue, Dillon, Harlen y Léna, 2012) y en problemas auténticos, favoreciendo así el aprendizaje significativo (Maturano, Aguilar, Núñez y Pereira, 2009). Este enfoque da la oportunidad de conseguir que los alumnos obtengan respuestas a través de los procesos mentales y procedimientos científicos (Furtak, 2006), por lo que favorece la familiarización con el trabajo científico (Caamaño, 2011) y la adquisición de competencias (Ferrés, Marbá y Sanmartí, 2015). La indagación se caracteriza por otorgar importancia a la curiosidad, fomentar la autonomía e integrar las dimensiones afectiva, conductual y cognitiva, incidiendo en la motivación (García y Ladino, 2008).

La introducción de esta metodología está apoyada por numerosos estudios que recogen los beneficios que presenta a nivel pedagógico. En primer lugar, en el artículo de Linn et al. (2014), se indica que este tipo de actividades motivan al alumnado incidiendo en los aspectos actitudinales cara la materia de ciencias. Cristobal y García (2013) señalan que a través de esta metodología se consigue el desarrollo de destrezas y habilidades para la elaboración del conocimiento científico. Otros autores como Akerson y Hanuscin (2007) indican que la indagación guiada es un método que favorece un acercamiento a aspectos relacionados con la naturaleza de la ciencia como el uso de pruebas para la justificación de afirmaciones o la importancia de la imaginación y la creatividad en el ámbito de la ciencia. 
Dado que de forma general las investigaciones muestran que la enseñanza a través de la indagación produce resultados positivos, el objetivo de este estudio es analizar los desempeños del alumnado durante la realización de una actividad de indagación guiada en el laboratorio de física sobre la flotabilidad de los cuerpos.

\section{Método}

La metodología de este estudio se enmarca en la investigación cualitativa, que se basa en el reconocimiento y análisis de distintas perspectivas a través de la reflexión del investigador como parte del proceso de producción de conocimiento en base a los datos recogidos durante la investigación y a la conducta observable (Flick, 2009). Según Corbin y Strauss (2008), este término se refiere a cualquier tipo de investigación que produzca resultados a los que no se llega por medio de procedimientos estadísticos u otros medios cuantificables.

\section{Participantes y contexto}

La investigación se lleva a cabo en un Instituto de Educación Secundaria urbano. Los grupos participantes en el estudio son dos clases de $2^{\circ}$ de ESO (A y B), con 17 y 20 alumnos y alumnas respectivamente que cursan la materia de Ciencias Naturales. Durante este curso, los y las estudiantes cursaron la materia correspondiente a la parte de biología, geología y química, por lo que sus experiencias de laboratorio previas se reducen a estos campos, siendo la actividad analizada en este artículo su primer contacto con la física en el laboratorio.

\section{Diseño de la investigación}

La investigación consta de una actividad que consiste en investigar los factores determinantes de la flotabilidad de un cuerpo. Dicha tarea se contextualiza en una salida que tendría lugar la semana siguiente en la cual los y las estudiantes participarían en un campamento de aventuras en el cual realizarían ciertas actividades acuáticas. En base a esto la tarea propuesta consiste diseñar y poner en práctica un dispositivo que permita averiguar si la posición de una persona y la forma de la canoa son determinantes para hundirnos o no practicando kayak. El enunciado de la actividad se reproduce en el anexo.

Para facilitarle al alumnado la resolución de la tarea, se le proporcionan una serie de cuestiones a resolver a modo de andamiaje, constituyendo un guión que posteriormente fue recogido para su análisis.

\section{Toma de datos y análisis}

Dado que lo que se pretende en este estudio es describir la situación observada con la realización de la actividad de indagación, las técnicas de recogida de datos escogidas son los guiones cumplimentados por el alumnado y las observaciones visuales llevadas a cabo durante el desarrollo de la sesión.

Cuando hablamos del análisis de datos en una investigación cualitativa hablamos de someter el texto a múltiples manipulaciones a través de un proceso de selección, categorización, comparación, validación e interpretación de la realidad con la finalidad de encontrar un sentido a la información (Guzmán, Cabrera, Yanes y Castro, 2008).

Para llevar a cabo el análisis de los datos obtenidos en esta indagación se elaboró una respuesta de referencia en la cual se basa la elaboración de una rúbrica en la que se 
recogen cinco dimensiones que comprenden los trazos generales de la indagación (NRC, 2000), con el fin de analizar los datos obtenidos durante el desarrollo del estudio.

\section{Las dimensiones incluidas son las siguientes:}

1) Hipótesis: Consiste en la realización de predicciones que respondan a la pregunta postulada en la indagación. Se consideran apropiadas las respuestas que contemplen si creen que la plastilina flotará o se hundirá y los factores que lo condicionan.

2) Diseño del procedimiento: Elaboración de la secuencia de experiencias que realizará el alumno para comprobar su hipótesis. Se considera que la respuesta es adecuada si incluía las fases de la investigación de forma clara y detallada.

3) Control de variables: En este paso se pretende determinar aquellos aspectos del experimento que se van a mantener invariables, constantes, y aquellos que van a variar, variables. En la rúbrica se considera como respuesta correcta no solo el reconocimiento de las variables sino también su correcta clasificación.

4) Interpretación de resultados: En este apartado se busca analizar los resultados obtenidos de la experimentación. Se considera correcta la respuesta que tiene en cuenta los dos aspectos propuestos en la investigación y no solo parte de ellos.

5) Conclusiones. Se elaboran partiendo de la interpretación de resultados y de la comprobación de las hipótesis. Para evaluar este apartado se considera como respuesta correcta aquella en la que se elabora una conclusión que incluya una explicación que dé respuesta al problema propuesto.

\section{Resultados}

Los resultados obtenidos se analizan de forma global para todo el grupo participante y a continuación de forma separada para cada una de las clases. Dado que durante el transcurso de la indagación el alumnado trabajó en pequeños grupos se analizan las respuestas para cada uno de estos grupos en relación al modelo de referencia. En la tabla 2 se reflejan los desempeños del alumnado a través de la frecuencia de respuestas que se adecúan a la ideal.

Tabla 2. Resultados del alumnado para las cinco dimensiones de la rúbrica

\begin{tabular}{|c|c|c|c|c|}
\hline Operación & Descripción & $2^{\circ} \mathrm{A}$ & $2^{\circ} \mathrm{B}$ & Total \\
\hline \multirow{3}{*}{ Hipótesis } & Elabora una hipótesis justificada & 5 & 4 & 9 \\
\hline & No justifica su hipótesis & 1 & 1 & 2 \\
\hline & Respuesta en blanco & 0 & 1 & 1 \\
\hline \multirow{3}{*}{ Procedimiento } & Detalla los pasos a seguir en la investigación & 3 & 6 & 9 \\
\hline & Los pasos son confusos o poco precisos & 3 & 0 & 3 \\
\hline & Respuesta en blanco & 0 & 0 & 0 \\
\hline \multirow{4}{*}{$\begin{array}{l}\text { Control } \\
\text { variables }\end{array}$} & $\begin{array}{l}\text { Identifica correctamente los factores constantes y } \\
\text { variables }\end{array}$ & 3 & 1 & 4 \\
\hline & Identifica correctamente los factores del experimento & 1 & 2 & 3 \\
\hline & No identifica los factores del experimento & 2 & 3 & 5 \\
\hline & Respuesta en blanco & 0 & 0 & 0 \\
\hline \multirow{3}{*}{$\begin{array}{l}\text { Interpretación } \\
\text { de resultados }\end{array}$} & $\begin{array}{l}\text { Refleja en los resultados los dos puntos solicitados } \\
\text { en el enunciado }\end{array}$ & 1 & 1 & 2 \\
\hline & $\begin{array}{l}\text { Refleja en los resultados uno de los puntos } \\
\text { solicitados en el enunciado }\end{array}$ & 5 & 5 & 10 \\
\hline & Respuesta en blanco & 0 & 0 & 0 \\
\hline
\end{tabular}




\begin{tabular}{llllll}
\hline & Elaboran una conclusión completa y adecuada & 1 & 0 & 1 \\
Conclusiones & Elaboran una conclusión incompleta o incorrecta & 3 & 4 & 7 \\
& $\begin{array}{l}\text { Repiten los resultados en el apartado de } \\
\text { conclusiones } \\
\text { Respuesta en blanco }\end{array}$ & & 1 & 3 \\
& & 0 & 1 & 1 \\
\hline
\end{tabular}

En lo referido a la dimensión hipótesis, se puede observar que las frecuencias más altas se sitúan en el nivel máximo de corrección para ambas clases, "Elabora una hipótesis justificada", coincidiendo con la tendencia del total de grupos. La mayoría de los grupos encuadrados en esta categoría refieren una hipótesis similar, como por ejemplo la formulada por el grupo A2 "Según las formas que le demos puede hundirse o no", estos grupos proponen una hipótesis en la que no solo reflejan si consideran que la plastilina se hundirá o no, sino que incluyen también el motivo por el que creen que esto sucede.

En lo que se refiere a la categoría "No justifica su hipótesis" encontramos que un grupo de alumnos en cada clase da una respuesta de este tipo, como por ejemplo el grupo A1 cuya respuesta es "Que no se va a hundir". En este caso los grupos solamente señalan si creen que la plastilina se hundirá o no, sin ningún tipo de justificación ni condicionante de este hecho.

Por último, hay un único grupo en el total de las dos clases que deja esta respuesta en blanco. Basándonos en las notas tomadas en clase, esta respuesta en blanco es debida a la consideración por parte de este grupo de que la elaboración de una hipótesis "no aporta nada a su investigación".

En general, no existen grandes diferencias entre ambas clases para esta dimensión. En la clase A, cinco de los seis grupos elaboran una hipótesis justificada y en la clase $B$ cuatro de seis lo hace. Hay además en ambas clases un grupo que no justifica su hipótesis. En cuanto a la respuesta en blanco solo se identifica un grupo en la clase B que se encuadre en esta categoría, este hecho indica que el alumnado se encontraba interesado e implicado en la resolución de la tarea.

La segunda dimensión de la rúbrica se corresponde con el procedimiento, en ella se analiza la corrección de la elaboración del procedimiento a seguir para comprobar la hipótesis propuesta en el primer punto del guion.

Se aprecia una notable diferencia entre las respuestas del grupo $B$, correctas en la totalidad de los grupos, seis de seis, y en el grupo $A$, que se reparten entre la respuesta correcta y la incorrecta, tres grupos para cada categoría, sin que haya ningún grupo que deje en blanco este apartado.

Entre los nueve grupos que elaboran un procedimiento correcto encontramos respuestas como la del grupo $\mathrm{A6}$ :

1. Echar agua en la botella

2. Hacer una bola con plastilina y comprobar si flota

3. Darle forma de canoa a la plastilina e introducirla en el agua

4. Hacer un churro con plastilina, ponerlo con forma de persona y introducirlo en el agua

En este grupo la respuesta, aunque poco precisa es adecuada para resolver la actividad ya que indica claramente, aunque de forma muy genérica, los pasos que van 
a seguir para resolver el problema y estos responde a todas las cuestiones propuestas en el mismo.

Para aquellos grupos que elaboran una respuesta confusa o poco precisa podemos utilizar como ejemplo la respuesta del grupo A3:

1. Cogemos el recipiente. En este caso es una botella. La llenamos de agua

2. Cogemos la plastilina y le hacemos forma de canoa

3. La metemos en agua y miramos si flota

4. Si flota con un peso o dedo vamos poniéndola en varios sitios para ver si volcaría, se hundiría o flotaría. También le podemos llenar el agujero con agua para ver si se hunde

5. Cogemos los resultados que concluimos

Aunque en este grupo el procedimiento incluye un mayor número de pasos que en el ejemplo anterior, se centra en un único apartado del enunciado del problema sin comprobar si el material flota independientemente de la forma ni si la posición puede influir. Además, su cuarto paso resulta confuso e irrelevante para la resolución del problema propuesto.

En cuanto a la dimensión "Control de variables", se establecen cuatro categorías para el análisis de la misma. Se puede observar una notable diferencia entre el grupo A y el $B$. En el primero hay un mayor número de alumnos que elaboran una respuesta aceptada, tres de seis, mientras que en el grupo B solo los alumnos de un equipo consiguieron una respuesta que se aproximara a la ideal. Todas las respuestas de esta dimensión encuadradas en esta categoría fueron similares o idénticas a la del grupo A4, "Constante: Agua, cantidad de plastilina, recipiente. Variable: Forma".

Sin embargo, existen grupos como el B2, cuya respuesta fue "Constante: Recipiente, volumen de agua. Variado: Forma de la plastilina, masa de la plastilina". Estos grupos identifican correctamente los factores a tener en cuenta en el experimento, pero no determinan cuáles de ellos se deben mantener constantes y cuales es necesario variar para el propósito de la indagación. Esta confusión indica que no identifican claramente la variable relevante para el estudio y qué otras se deben mantener constantes para poder analizar la importancia de la variación de la primera.

Por último, encontramos aquellos grupos que no reconocen los aspectos a tener en cuenta para la realización del experimento. Un ejemplo de esto lo constituye el grupo A6, que considera "Constante: Agua en la botella, botella. Variable: Forma de la plastilina", obviando en esta clasificación la masa de plastilina utilizada.

Para el análisis de la dimensión "Interpretación de resultados" en la rúbrica se comprenden tres categorías de análisis. Se observa que los resultados son idénticos en las dos clases participantes en el desarrollo de esta propuesta. Ninguno de los grupos deja esta respuesta en blanco. Solo un grupo en cada aula consigue responderla de forma totalmente correcta, como ejemplo se toma el grupo A6:

1. La plastilina no flota

2. La plastilina con forma de canoa flota y aguanta con ocupantes

3. La plastilina con forma de persona de pie no flota. La plastilina con forma de muerto sí que flota 
Se considera correcta esta respuesta porque, aunque de forma breve, recoge resultados sobre todos los puntos que se proponía investigar en el enunciado que se aportó

El resto de los grupos, cinco de cada clase, responden de forma incompleta, todos ellos por obviar la primera parte de la indagación propuesta. Un ejemplo representativo de las respuestas encuadradas en esta categoría lo aporta el grupo A1 que responde "De por sí no flota, pero en cachos muy pequeños sí. Si pones un cacho con forma de kayak sí que flota. Es importante la forma". En este grupo se obvia completamente lo referente al condicionamiento de la flotabilidad debido a la posición. En otros grupos encuadrados en esta misma categoría no solo no se contempla la influencia de la posición, sino que tampoco se hace referencia a la flotabilidad asociada a la naturaleza del material empleado, un ejemplo es el grupo A3 que indica que "Metemos la barca pero no flotaba, entonces aumentamos los laterales y doblamos algo la parte de delante y atrás, entonces no se hunde porque no da entrado el agua por ningún lado"

Por último, se encuentra la dimensión "'Conclusiones" para el análisis de la cual se proponen cuatro categorías. Se observa que la mayoría de los alumnos de ambas clases elaboran una conclusión que se encuentra incompleta o es incorrecta. Como ejemplo de esto tomamos la conclusión elaborada por el grupo A1 "Flota con un kayak de plastilina por su tamaño". Se observa que esta conclusión no se relaciona con ninguno de los aspectos propuestos en el problema, por lo que se puede asociar este error con una comprensión lectora insuficiente.

El siguiente nivel con un mayor número de grupos es el de aquellos que utilizan el apartado de conclusiones para volver e reflejar los resultados, como es el caso del grupo B1 que responde a este apartado indicando que "Flota con forma de medio coco". En el caso de estos grupos, su distanciamiento de la respuesta ideal obedece a una incomprensión de la diferencia entre resultados y conclusiones.

Por último, un único grupo de la totalidad de las clases consigue elaborar una conclusión completa, el grupo A6 que indica que "La forma de plastilina sí que influye, la plastilina sin forma se hunde, con forma de canoa no se hunde y con forma de persona de pie sí que se hunde mientras que con forma de muerto no se hunde". Este grupo sí indica el factor condicionante de la flotabilidad en su conclusión.

En resumen, los alumnos proporcionan un elevado número de respuestas coincidentes con el ideal en la dimensión "hipótesis", hecho que puede estar justificado en la explicación que se les dio sobre este aspecto durante el desarrollo de la sesión, que consistió en definir la hipótesis como una predicción, una opinión de lo que ellos consideran que va a ocurrir y por qué.

En contraposición desciende notablemente el número de respuestas coincidentes con el ideal en la dimensión "control de variables". Este hecho puede estar debido a una especial complicación de este punto, situación que coincide con otros estudios como el de Ferrés, Marbá y Sanmartí (2015). Además existen diferencias importantes entre las respuestas dadas por el grupo $\mathrm{A}$, en el que tres agrupaciones de trabajo dan una respuesta coincidente con el ideal, y las del grupo $B$, en el que solo una agrupación da una respuesta que coincide con el ideal.

Los números más reducidos de respuestas coincidentes con la ideal se encuentra para las dimensiones "Interpretación de resultados" y "Conclusiones", siendo la primera un grupo de cada clase los que consiguieron la respuesta ideal y la segunda 
solo un grupo de una de las clases. Esta situación puede estar justificada en la inexperiencia del alumnado en la realización de tareas de indagación guiada y en la falta de explicación de las mismas en el guion que se les aportó.

Por último, se observar diferencias significativas entre los grupos en la dimensión de "Procedimiento", en la que el total de alumnos del grupo B da una respuesta coincidente con la ideal mientras que el grupo A reparte sus respuestas a partes iguales entre la ideal y una incompleta. Tomando como base las observaciones recogidas durante el desarrollo de esta sesión se podría justificar esta diferencia en el mayor intercambio de información entre los grupos de trabajo producido en el grupo B.

Para realizar una observación global de las respuestas del alumnado se elaboró la figura 1, que toma como datos el total de respuestas coincidentes con la ideal, el total de respuestas en blanco y aquellas que se alejan del ideal para cada grupo de participantes en este estudio.

Se observa que la clase que más se aproxima al ideal sería $2^{\circ} \mathrm{A}$ por presentar no solo un mayor número de respuestas coincidentes con la ideal sino también un menor número de respuestas en blanco.
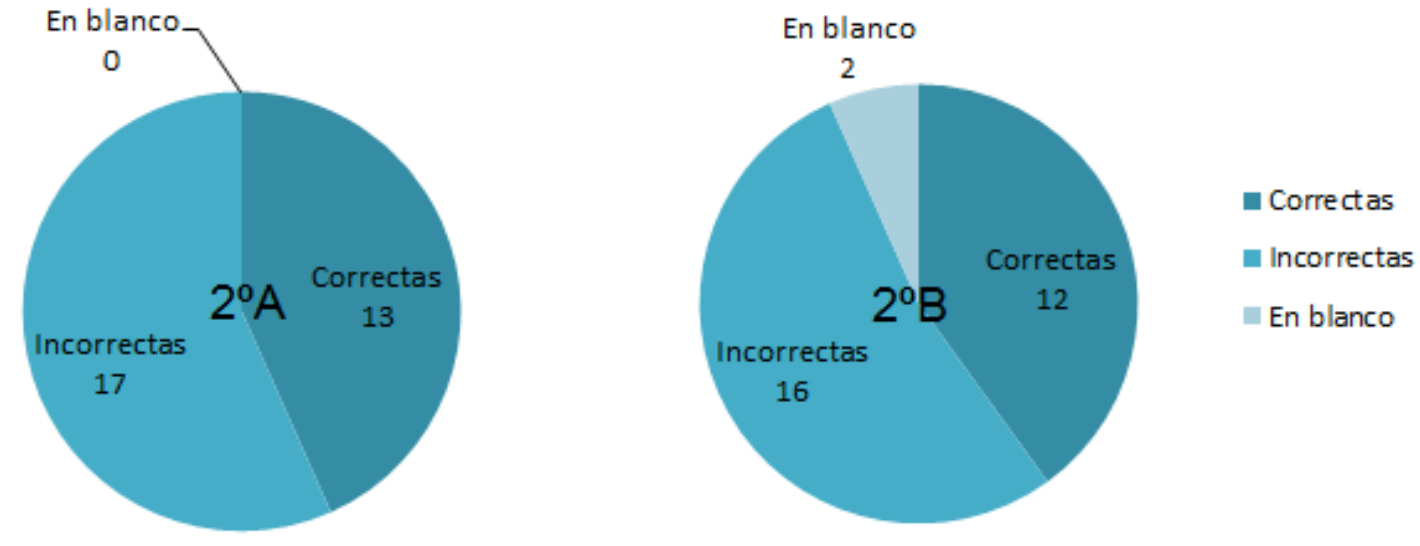

Figura 1. Representación de las respuestas para el global de cada clase.

\section{Discusión y conclusiones}

El objetivo de esta investigación es analizar los desempeños del alumnado durante la realización de una actividad de indagación en el laboratorio. Para llevar a cabo este análisis se elaboró una rúbrica que comprende cinco dimensiones relativas a las operaciones de indagación (NRC, 2000), las cuales presentan diversas categorías en función de la mayor o menor adecuación al modelo de referencia para resolver la tarea.

Los resultados demuestran que existen diferencias en la mayor o menor adecuación de los desempeños del alumnado al modelo de referencia en ambas clases. A nivel general existen algunos alumnos y alumnas que se ajustan más a dicho modelo que otros, que se encuentran más distanciados del ideal.

Artículos como el Krajcik et al. (1998) apoyan que, incluso en los primeros contactos con este tipo de experiencias, los estudiantes tienen la capacidad de elaborar diseños, más o menos sofisticados, que les permiten obtener datos para responder sus preguntas, tal y como sucedió en esta experiencia. 
Los resultados más destacados sugieren que existen ciertos procedimientos de la experiencia de indagación que entrañan una mayor dificultad para el alumnado.

En primer lugar, tal como refiere Barrow (2006), se observa que la implementación de la indagación en el aula requiere un mayor tiempo de aprendizaje debido a que el alumnado tiene que adaptarse a una metodología más activa y aportar sus explicaciones y habilidades. Esto se ve reflejado en el hecho de que parte de los alumnos requirieron algo más de tiempo al finalizar la clase para acabar de recoger los resultados de su experiencia.

Además de esta dificultad, se observa y está apoyado por artículos como el de Ferrés, Marbá y Sanmartí, (2015), que existen otras en la formulación de preguntas, en el formato de la elaboración hipótesis, en el diseño del experimento y en la recogida de datos y generación de conclusiones. Tras llevar a cabo esta experiencia se corroboró este hecho, ya que estas últimas son las tres dimensiones de la rúbrica con un menor número de respuestas coincidentes con el ideal.

Esta actividad pretendía partir de los conocimientos e ideas previas del alumnado para que estos fueran usados como hipótesis a corroborar a través de la utilización de la metodología científica, constituyendo el problema propuesta una experiencia que entre en conflicto con las ideas alternativas para favorecer así el cambio conceptual.

Se observó que en la mayoría de los casos la ideas previas efectivamente funcionan como hipótesis y que, en aquellos casos en los que existen ideas alternativas con las que la experiencia entra en conflicto, hay algunos en los que el alumnado rechaza su idea previa, pero en la mayoría de los mismos existe una tendencia a desestimar los datos que contrarían su idea, tal y como indican Hierrezuelo y Montero (1989).

Esta tendencia a mantener las ideas previas pone de relevancia la necesidad de fomentar el debate tras llevar a cabo este tipo de experiencias, señalada por Driver, Guesne y Tiberghien (1989) o incluso la posterior realización de nuevas experiencias relacionadas con ideas alternativas que persistan, para así asegurar que el cambio conceptual y la asimilación de la idea aceptada científicamente tiene lugar.

Sin embargo, a pesar de las dificultades que la implementación de esta metodología puede suponer, existen estudios, como el de Rissing y Cogan (2009) que demuestran que los estudiantes que llevan a cabo indagaciones en lugar de protocolos de laboratorio estándar muestran una mejora significativa en la asimilación de conocimiento y en la seguridad en las técnicas e información utilizadas.

En lo que se refiere a las implicaciones educativas que podemos extraer de este estudio se concluye a través de la observación del desarrollo de la práctica y de los resultados obtenidos que una aproximación a la materia a través de una indagación guiada introduce aspectos de la metodología científica que no están presentes en la enseñanza alejada de las experiencias de laboratorio. Ya que, al ser esta su primera experiencia con actividades de este tipo, los alumnos presentan problemas para responder ciertas cuestiones de la indagación.

Además, también podemos extraer que este tipo de experiencias promueven el interés de los estudiantes y son fuente de motivación cara la materia, tal como apoya Tortosa (2013), lo que favorece el aprendizaje significativo y el desarrollo de la competencia científica en mayor medida que una metodología de enseñanza más teórica. 
Por último, en el artículo de Krajcik et al. (1998) se refiere que las sugerencias y preguntas del profesor resultan cruciales para llevar a los alumnos a reflexionar sobre los aspectos importantes de su investigación, distanciándolos de la focalización en los aspectos procedimentales. Esta afirmación se corroboró en el transcurso de la realización de la actividad en el laboratorio, durante la cual se le formularon preguntas a los distintos grupos en aquellos momentos en los que les resultaba complicado orientar su investigación, tales como "Si realizas este procedimiento, ¿cómo sabes si el hecho de que tu embarcación flote no es porque la plastilina flota en el agua?". Con este tipo de preguntas también se les pone de relevancia a los alumnos la necesidad de elaborar controles (Krajcik et al., 1998).

Durante el desarrollo y planificación de la actividad se encontraron algunas dificultades. En primer lugar están aquellas que se relacionan con la falta de tiempo para introducir experiencias de este tipo debido a la saturación de contenidos, referida en el artículo de Miralles, Maquilón, Hernández y García (2012). Además dentro de las dificultades que se incluyen en aquellas relacionadas con el centro y su entorno, se encontró la que se refiere a los recursos y materiales de los que dispone el profesorado, ya que fue necesario aportar parte del material requerido para la práctica.

Para finalizar es preciso señalar que se necesitan medidas para intentar minimizar los obstáculos surgidos con la introducción de actividades innovadoras en el aula o laboratorio, como la indagación que se presentan en este trabajo, entre las cuales se necesitaría mejorar la formación inicial y permanente del profesorado, así como los procesos de comunicación entre el profesorado y la cooperación de los centros con las universidades, tal y como proponen Miralles et al. (2012).

Referencias

Akerson, V.L. y Hanuscin, D.L. (2007). Teaching the nature of science through inquiry: The results of a three-year professional development program. Journal of Research in Science Teaching, 44(5), 653-680.

Álvarez, M., Arias, A., Pérez, U. y Serrallé, J. F. (2013). La historia de las ciencias en el desarrollo de competencias científicas. Enseñanza de las Ciencias, 31(1), 213-233.

Anderson, R.D. (2002). Reforming Science Teaching: What Research says about Inquiry. Journal of Science Teacher Education, 13(1), 1-12.

Artigue, M., Dillon, J., Harlen, W., y Léna, P. (2012). Learning through Inquiry: Background Resources for implementing Inquiry in Science and Mathematics at School. Francia: Proxecto Fibonacci. Recuperado o 4 de xuño de 2016 de http://www.fibonacci-project.eu

Barrow, L.H. (2006). A Brief History of Inquiry: From Dewey to Standards. Journal of Science Teacher Education, 17, 265-278.

Bell, R., Smetana, L. y Binns I. Simplifying inquiry instruction. The Science Teacher, 72, 30-34

Caamaño, A. (2011). La investigación escolar es la actividad que mejor integra el aprendizaje de los diferentes procedimientos científicos. En E. Pedrinaci, (coord.). 11 ideas clave. El desarrollo de la competencia científica (127-146). Barcelona: Graó.

Corbin, J. y Strauss A. (2008). Basics of Qualitative Research: Techniques and Procedures for Developing Grounded Theory. Los Ángeles, CA: Sage Publications Inc.

Cortel, A. (Coord.), Calvo, A., Carreras, C., Carrión, J.A., García, J.J., León, J.J., Pastor, J.M., Tornos, J. y Yuste, M. (2004). Nuevos enfoques para la enseñanza física. Madrid: Secretaría General Técnica. Subdirección General de Información y Publicaciones.

Cristobal, C.M. y García, H.A. (2013). La indagación científica para la enseñanza de las ciencias. Horizonte de la ciencia, 3(5), 99-104. 
Driver, R., Guesne, E. y Tiberghien A. (1989). Ideas cientificas en la infancia y la adolescencia. Madrid: Ediciones Morata, S.A. e Centro de Publicaciones del Ministerio de educación y Ciencia.

Ferrés, C., Marbà, A. y Sanmartí, N. (2015). Trabajos de indagación de los alumnos: instrumentos de evaluación e identificación de dificultades. Revista Eureka sobre Enseñanza y Divulgación de las Ciencias, 12(1), 22-37.

Flick, U. (2009). An introduction to qualitative research. Nueva Delhi: SAGE Publications India Pvt Ltd.

Furtak, E.M. (2006). The problem with answers: an exploration of guided scientific inquiry teaching. Science Education, 90(3), 453-467.

García-Carmona, A. (2009). Investigación en didáctica de la Física: tendencias actuales e incidencia en la formación del profesorado. Latin-American Journal of Physics Education, 3(2), 369-375.

García-Carmona, A. (2011). Aprender Física y Química mediante secuencias de enseñanza investigadoras. Fundamentación y propuestas para Educación Secundaria Obligatoria. Málaga: Ediciones Aljibe, S.L.

García, G.A. y Ladino, Y. (2008). Desarrollo de competencias científicas a través de una estrategia de enseñanza y aprendizaje por investigación. Studiositas, 3(3), 7-16.

Guzmán, R., Cabrera, L., Yanes, J. y Castro, J. (2008). Análisis de la investigación cualitativa en educación desarrollada en el Estado Español. Revista Qurriculum, 21, 157-184.

Hofstein, A. y Lunetta, V.N. (2003). The Laboratory in Science Education: Foundations for the Twenty First Century. Science education, 88(1), 28-54.

Hierrezuelo, J. y Montero A. (1989). La ciencia de los alumnos. Su utilización en la didáctica de la Física y la Química. Barcelona: Centro de Publicaciones del MEC y Editorial Laia, S.A.

Krajcik, J., Blumenfeld, P.C., Marx, R.W., Bass, K.M, Fredricks, J y Solloway, E. (1998). Inquiry in Project-Based Science Classrooms: Initial Attempts by Middle School Students. The journal of the learning sciences, 7(3 e 4), 313-350.

Linn, M.C., Gerard, L., Ryoo, K., McElhaney, K. Liu, O.L. y Rafferty, A.N. (2014). Computer-Guided Inquiry to Improve Science Learning. Science, 344, 155-156.

Maturano, C., Aguilar, S., Núñez, G. y Pereira, R. (2009). Una experiencia propuesta como tarea de investigación en enseñanza secundaria sobre las condiciones de flotabilidad de una embarcación. Latin-American Journal of Physics Education, 3(1), 380-387.

Miralles, P., Maquilón, J.J., Hernández, F. y García, A. (2012). Dificultades de las prácticas docentes de innovación educativa y sugerencias para su desarrollo. Revista Electrónica Interuniversitaria de Formación del Profesorado, 15(1), 19-26.

National Research Council. (2000). Inquiry and the National Science Education Standards. Washington, D.C.: National Academy Press.

Rissing, S.W. y Cogan, J.G. (2009). Can an Inquiry Approach Improve College Student Learning in a Teaching Laboratory?. Life Sciences Education, 8, 55-61.

Tortosa, M. (2013). Aprendizaje sobre disoluciones reguladoras de pH mediante indagación guiada utilizando sensores. Enseñanza de las ciencias, 31(1), 189-211.

Windschitl, M. (2003). Inquiry Projects in Science Teacher Education: What Can Investigative Experiences Reveal About Teacher Thinking and Eventual Classroom Practice. Science Teacher Education, 87, 112.143. 


\section{Anexo: Guión de la tarea proporcionado a los estudiantes}

Los alumnos de $2 \%$ del centro vais a hacer una salida a los Peares la semana que viene. Con el fin de estar preparados para hacer kayak queremos determinar si nuestra posición influye en nuestra capacidad para no hundirnos y si la forma de la canoa es importante para que podamos montar en ella sin hundirnos.

Para hacer esto disponemos de plastilina, agua y un recipiente.

¿Cómo podríamos averiguarlo?

¿Qué pasos vamos a seguir?

¿Qué crees que va a pasar?

¿Qué vamos a mantener constante y que vamos a variar?

\begin{tabular}{|l|l}
\hline Constante & Variable \\
\hline
\end{tabular}

Resultados

Conclusiones 